\title{
Theory of electrokinetic phenomena in fibrous porous media caused by gradients of electrolyte concentration
}

\author{
Yeu K. Wei, Huan J. Keh* \\ Department of Chemical Engineering, National Taiwan University, Taipei 106-17, Taiwan, ROC
}

\begin{abstract}
The steady diffusioosmotic flow of an electrolyte solution in the fibrous medium constructed by an ordered array of parallel charged circular cylinders is analytically studied. The prescribed electrolyte concentration gradient is constant but can be oriented arbitrarily with respect to the axes of the cylinders. The electric double layer surrounding each cylinder may have an arbitrary thickness relative to the radius of the cylinder. A unit cell model, which allows for the overlap of the double layers of adjacent cylinders, is employed to account for the effect of fibers on each other. The electrostatic potential distribution in the fluid phase of a cell is obtained by solving the linearized Poisson-Boltzmann equation, which applies to the case of low surface potential or low surface charge density of the cylinders. An analytical formula for the diffusioosmotic/electroosmotic velocity of the electrolyte solution as a function of the porosity of the array of cylinders correct to the second order of their surface charge density or zeta potential is derived as the solution of a modified Stokes equation. In the absence of a macroscopic electric field induced by the electrolyte gradient (or externally imposed), the fluid flows (due to the chemiosmotic contribution) toward lower electrolyte concentration. With an induced electric field, competition between electroosmosis and chemiosmosis can result in more than one reversal in direction of the fluid flow over a small range of the fiber surface potential. In the limit of maximum porosity, these results can be interpreted as the diffusiophoretic and electrophoretic velocities of an isolated circular cylinder caused by the applied electrolyte gradient or electric field.
\end{abstract}

(C) 2003 Elsevier Science B.V. All rights reserved.

Keywords: Diffusioosmosis; Electroosmosis; Diffusiophoresis; Electrophoresis; Charged cylinder; Unit cell model

\section{Introduction}

The flow of electrolyte solutions in a small pore with a charged wall is of much fundamental and practical interest in various areas of science and engineering. In general, driving forces for this

* Corresponding author. Tel.: +886-2-2363-5462; fax: + 886-2-2362-3040.

E-mail address: huan@ccms.ntu.edu.tw (H.J. Keh). electrokinetic flow include dynamic pressure differences between the two ends of the pore (a streaming potential is developed as a result of zero net electric current) and tangential electric fields that interact with the electric double layer adjacent to the pore wall (electroosmosis). Problems of fluid flow in porous media caused by these well-known driving forces were studied extensively in the past century [1-6].

Another driving force for the electrokinetic flow in a micropore, which has commanded less atten- 
tion, involves tangential concentration gradients of an ionic solute that interacts with the charged pore wall. This solute-wall interaction is electrostatic in nature and its range is the Debye screening length $\kappa^{-1}$. The fluid motion associated with this mechanism, known as "diffusioosmosis" (which is the complementary transport phenomenon to the diffusiophoresis of colloidal particles in prescribed solute concentration gradients), has been discussed mainly for solutions near a plane wall [4,7-9]. Electrolyte solutions with a concentration gradient of order $1 \mathrm{M} \mathrm{cm}^{-1}$ along rigid surfaces with a zeta potential of order kT/e $(\sim 25$ $\mathrm{mV} ; e$ is the charge of a proton, $k$ is the Boltzmann constant, and $T$ is the absolute temperature) can flow by diffusioosmosis at rates of several microns per second.

The analytical expression for the diffusioosmotic velocity of electrolyte solutions parallel to a charged plane wall [7] can be applied to the corresponding flow in capillary tubes and slits when the thickness of the double layer adjacent to the capillary wall is small compared with the capillary radius. However, in some practical applications involving dilute electrolyte solutions in very fine pores, this condition is no longer satisfied and the dependence of the fluid flow on the electrokinetic radius $\kappa R$, where $R$ is the radius of a capillary tube or the half thickness of a capillary slit, must be taken into account. Recently, the diffusioosmosis of electrolyte solutions in a capillary tube or slit with an arbitrary value of $\kappa R$ was analyzed [10] for the case of small surface potential or surface charge density at the capillary wall. Closed-form formulas for the fluid velocity profile and average fluid velocity on the cross section of the capillary tube or slit were derived.

The capillary model of porous media is not realistic for either granular or fibrous systems, for it does not allow for the convergence and divergence of flow channels. For electrokinetic flow within beds of particles or synthetic microporous membranes, it is usually necessary to account for the effects of pore geometry, tortuosity, etc. To avoid the difficulty of the complex geometry appearing in beds of particles, a unit cell model [11-13] was often employed to predict these effects on the relative motions between a granular bed and the bulk fluid. This model involves the concept that a bed of identical particles can be divided into a number of identical cells, one particle occupying each cell at its center. The boundary value problem for multiple particles is thus reduced to the consideration of the behavior of a single particle and its bounding envelope. In the past three decades, the unit cell model was used by many researchers to predict various electrokinetic properties such as the mean electrophoretic mobility [14-22], effective electric conductivity [17,20-23], mean sedimentation rate [24-27], and sedimentation potential [24-28] of a suspension of charged spherical particles as well as the transverse electroosmotic mobility of electrolyte solutions in a fibrous porous medium [29-32]. Recently, using the cell model, the present authors derived analytical expressions for the mean diffusioosmotic/ diffusiophoretic velocity in a bed of dielectric spheres [33] and within an array of parallel charged circular cylinders caused by a transversely imposed electrolyte concentration gradient [34].

For a general case of diffusioosmosis/electroosmosis of a fluid solution in a fibrous system constructed by an array of parallel cylinders, the applied electrolyte concentration gradient can be taken as a combination of its transversal and longitudinal components with respect to the orientation of the cylinders. Hence, the problem can be divided into two, if it is linearized, and they might be separately solved. The overall diffusioosmotic/electroosmotic velocity of the bulk fluid can be obtained by the vectorial addition of the twocomponent results. Since the problem of the transverse diffusioosmotic flow in a homogeneous array of parallel charged circular cylinders has been solved in a previous paper [34], in this work we only need to treat the diffusioosmosis in the array generated by a longitudinal electrolyte gradient. The unit cell model is still used in the analysis. The electric potential at the cylinder surfaces (or the surface charge density) is assumed to be uniform and low, but no assumption is made as to the thickness of the electric double layers relative to the radius of the cylinders. A closedform expression for the diffusioosmotic/electroosmotic velocity profile in a cell is obtained in Eqs. (16), (17a) and (17b). The information provided by 
this work may prove relevant in understanding the chemotactic flow of fluids and transport of particles in physiological media.

\section{Analysis}

We consider the diffusioosmotic flow of a fluid solution of a symmetrically charged binary electrolyte of valence $Z$ (where $Z$ is a positive integer) through a uniform array of parallel, identical, circular cylinders at the steady state. The discrete nature of the surface charges, which are uniformly distributed on each cylinder, is neglected. The applied electrolyte concentration gradient $\nabla n^{\infty}$ is a constant along the axial $(z)$ direction of the cylinders, where $n^{\infty}(z)$ is the linear concentration distribution of the electrolyte in the bulk solution phase in equilibrium with the fluid inside the array. The electrolyte ions can diffuse freely in the fibrous porous medium, so there exists no regular osmotic flow of the solvent. As shown in Fig. 1, we employ a unit cell model in which each dielectric cylinder of radius $a$ is surrounded by a coaxial circular cylindrical shell of the fluid solution having an outer radius of $b$ such that the fluid/cell volume ratio is equal to the porosity $1-$ $\varphi$ of the fiber matrix; viz. $\varphi=(a / b)^{2}$. The cell as a whole is electrically neutral. It is assumed that $a \ll$ $L$ and $L\left|\nabla n^{\infty}\right| / n^{\infty}(0) \ll 1$, where $L$ is the length of the cylinder and $z=0$ is set at the midpoint along the cylinder. Thus, the variation of the electrostatic potential (excluding the macroscopic electric field induced by the electrolyte gradient) and ionic

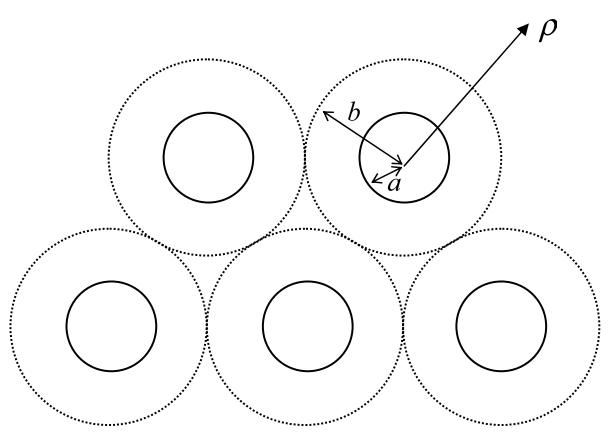

Fig. 1. Geometrical sketch of the unit cell model for a uniform array of identical parallel circular cylinders. concentrations in the electric double layer adjacent to the cylinder surface with the axial position can be ignored.

\subsection{Electrostatic potential distribution}

We first deal with the electric potential distribution in the fluid phase on a plane normal to the axis of the cylinder in a unit cell. If $\psi(\rho)$ represents the electrostatic potential at a point distance $\rho$ from the axis of the cylinder relative to that in the bulk solution and $n_{+}(\rho, z)$ and $n_{-}(\rho, z)$ denote the local concentrations of the cations and anions, respectively, then Poisson's equation gives:

$$
\begin{aligned}
& \frac{1}{\rho} \frac{\mathrm{d}}{\mathrm{d} \rho}\left(\rho \frac{\mathrm{d} \psi}{\mathrm{d} \rho}\right) \\
& \quad=-\frac{4 \pi Z e}{\varepsilon}\left[n_{+}(\rho, 0)-n_{-}(\rho, 0)\right] .
\end{aligned}
$$

In this equation, $\varepsilon=4 \pi \varepsilon_{0} \varepsilon_{\mathrm{r}}$, where $\varepsilon_{\mathrm{r}}$ is the relative permittivity of the electrolyte solution and $\varepsilon_{0}$ is the permittivity of a vacuum. The local ionic concentrations can also be related to the electrostatic potential by the Boltzmann equation,

$n_{ \pm}=n^{\infty} \exp \left(\mp \frac{Z e \psi}{k T}\right)$.

Substitution of Eq. (2) into Eq. (1) leads to the well-known Poisson-Boltzmann equation. For small values of $\psi$, the Poisson-Boltzmann equation can be linearized (known as the DebyeHuckel approximation), and Eq. (1) becomes:

$\frac{1}{\rho} \frac{\mathrm{d}}{\mathrm{d} \rho}\left(\rho \frac{\mathrm{d} \psi}{\mathrm{d} \rho}\right)=\kappa^{2} \psi$

where $\kappa$ is the reciprocal of the Debye screening length defined by:

$\kappa=\left[\frac{8 \pi Z^{2} e^{2} n^{\infty}(0)}{\varepsilon k T}\right]^{1 / 2}$.

The boundary conditions for $\psi$ are:

$\rho=a: \quad \psi=\zeta$,

$\rho=b: \quad \frac{\mathrm{d} \psi}{\mathrm{d} \rho}=0$, 
where $\zeta$ is the zeta potential of the cylinder surface (at the shear plane).

The solution to Eqs. (3), (5a) and (5b) is:

$\psi=\zeta \frac{I_{1}(\kappa b) K_{0}(\kappa \rho)+K_{1}(\kappa b) I_{0}(\kappa \rho)}{I_{1}(\kappa b) K_{0}(\kappa a)+K_{1}(\kappa b) I_{0}(\kappa a)}$,

where $I_{n}$ and $K_{n}$ are the modified Bessel functions of the first and second kinds, respectively, of order $n$. Due to the linearization of the PoissonBoltzmann equation, the normalized potential distribution $\psi / \zeta$ is independent of the dimensionless parameter $\mathrm{Ze \zeta /kT}$.

If the surface charged density $\sigma$, instead of the surface potential $\zeta$, is known on the cylinder, the boundary condition specified by Eq. (5a) should be replaced by the Gauss condition,

$\rho=a: \quad \frac{\mathrm{d} \psi}{\mathrm{d} \rho}=-\frac{4 \pi \sigma}{\varepsilon}$.

The solution for $\psi$ given by Eq. (6) still holds for this condition, with the relation between $\zeta$ and $\sigma$ for arbitrary values of $\kappa a$ and $\kappa b$ as:

$\zeta=\frac{4 \pi \sigma}{\varepsilon \kappa} \frac{I_{1}(\kappa b) K_{0}(\kappa a)+K_{1}(\kappa b) I_{0}(\kappa a)}{I_{1}(\kappa b) K_{1}(\kappa a)-K_{1}(\kappa b) I_{1}(\kappa a)}$.

In the limit of the largest possible porosity of the fiber matrix (or $\varphi=(a / b)^{2}=0$ ), Eqs. (6) and (8) reduce to:

$\psi=\zeta \frac{K_{0}(\kappa \rho)}{K_{0}(\kappa a)}$

and

$\zeta=\frac{4 \pi \sigma}{\varepsilon \kappa} \frac{K_{0}(\kappa a)}{K_{1}(\kappa a)}$.

For a charged cylinder with a given radius, Eq. (10) predicts that $\zeta$ decreases with an increase in $\kappa a$ for the case of constant surface charge density and $\sigma$ increases with an increase in $\kappa a$ for the case of constant surface potential.

\subsection{Fluid velocity distribution}

We now consider the steady flow of an electrolyte solution through a unit cell under the influence of a constant concentration gradient $\nabla n^{\infty}$ of the electrolyte prescribed axially. The momentum balances on the Newtonian fluid in the $\rho$ and $z$ directions give:

$\frac{\partial p}{\partial \rho}+e Z\left(n_{+}-n_{-}\right) \frac{\mathrm{d} \psi}{\mathrm{d} \rho}=0$,

$\frac{\eta}{\rho} \frac{\mathrm{d}}{\mathrm{d} \rho}\left(\rho \frac{\mathrm{d} u}{\mathrm{~d} \rho}\right)=\frac{\partial p}{\partial z}-e Z\left(n_{+}-n_{-}\right) E$,

where $u(\rho)$ is the fluid velocity in the axial direction of increasing electrolyte concentration (satisfying the equation of continuity for an incompressible fluid), $p(\rho, z)$ is the pressure, and $\eta$ is the viscosity of the fluid. The macroscopic electric field $E$ in Eq. (11b) arises spontaneously due to the imposed concentration gradient of the electrolyte and the difference in mobilities of the cation and anion of the electrolyte. Under the condition that there is no electric current generated by the cocurrent diffusion of the electrolyte ions in an electrically neutral bulk solution, this induced electric field can be expressed as [4,5,35]:

$E=\frac{k T}{Z e} \beta \frac{\left|\nabla n^{\infty}\right|}{n^{\infty}(0)}$,

which is a constant, where:

$\beta=\frac{D_{+}-D_{-}}{D_{+}+D_{-}}$,

and $D_{+}$and $D_{-}$are the diffusion coefficients of the cation and anion, respectively. Equation (12) is correct for the electric field induced by the bulk concentration gradient of the electrolyte solution in the cell for the case with the Debye-Huckel approximation. When the effect of radial distributions of ions is considered for the local induced electric field, the variable $\beta$ in Eq. (12) should be replaced by $\beta+\left(\beta^{2}-1\right) Z e \psi / k T+\mathrm{O}\left(\psi^{2}\right)$.

The boundary conditions for $u$ are:

$\rho=a: \quad u=0$,

$\rho=b: \quad \frac{\mathrm{d} u}{\mathrm{~d} \rho}=0$.

After the substitution of Eq. (2) for $n_{ \pm}$into Eq. (11a) and the application of the Debye-Huckel approximation, the pressure distribution can be determined, with the result: 
$p=p_{0}+\frac{n^{\infty}}{k T}(Z e)^{2}\left\{[\psi(\rho)]^{2}-[\psi(b)]^{2}\right\}+\mathrm{O}\left(\zeta^{3}\right)$,

where $p_{0}$ is the pressure on the outer (virtual) boundary of the unit cell, which is a constant in the absence of applied pressure gradient. Substituting the ionic concentration distributions of Eq. (2), the pressure profile of Eq. (15), and the electric field of Eq. (12) into Eq. (11b) and solving for the fluid velocity distribution subject to the boundary conditions in Eqs. (14a) and (14b), we obtain:

$$
\begin{aligned}
u= & -\frac{2 Z e}{\eta \kappa^{2}}\left|\nabla n^{\infty}\right|\left[\beta \zeta \Theta_{1}\left(\kappa a, \frac{a}{b}, \frac{\rho}{a}\right)\right. \\
& \left.+\frac{Z e}{8 k T} \zeta^{2} \Theta_{2}\left(\kappa a, \frac{a}{b}, \frac{\rho}{a}\right)+\mathrm{O}\left(\zeta^{3}\right)\right],
\end{aligned}
$$

where $\Theta_{1}$ and $\Theta_{2}$ are functions given by:

$$
\begin{aligned}
\Theta_{1} & =\frac{I_{1}(\kappa b)\left[K_{0}(\kappa a)-K_{0}(\kappa \rho)\right]+K_{1}(\kappa b)\left[I_{0}(\kappa a)-I_{0}(\kappa \rho)\right]}{I_{1}(\kappa b) K_{0}(\kappa a)+K_{1}(\kappa b) I_{0}(\kappa a)} \\
& =1-\frac{\psi(\rho)}{\zeta} \\
\Theta_{2} & =4 \kappa^{2} \int_{\rho}^{a} \frac{1}{\rho} \int_{b}^{\rho} \rho\left\{\left[\frac{\psi(\rho)}{\zeta}\right]^{2}-\left[\frac{\psi(b)}{\zeta}\right]^{2}\right\} \mathrm{d} \rho \mathrm{d} \rho .
\end{aligned}
$$

Note that the diffusioosmotic velocity given by Eq. (16) at the position $\rho=b$ can also be viewed as the diffusiophoretic velocity (in the opposite direction) of the charged cylinder caused by the imposed electrolyte gradient.

The average (superficial) fluid velocity $\langle u\rangle$ over a cross section of the unit cell can be expressed as:

$$
\begin{aligned}
\langle u\rangle= & -\frac{2 Z e}{\eta \kappa^{2}}\left|\nabla n^{\infty}\right|\left[\beta \zeta\left\langle\Theta_{1}\right\rangle+\frac{Z e}{8 k T} \zeta^{2}\left\langle\Theta_{2}\right\rangle\right. \\
& \left.+\mathrm{O}\left(\zeta^{3}\right)\right] .
\end{aligned}
$$

Here, the definition of the angle brackets is:

$\langle u\rangle=\frac{2}{b^{2}} \int_{a}^{b} u(\rho) \rho d \rho$.

It can be found that:

$\left\langle\Theta_{1}\right\rangle=\frac{I_{1}(\kappa b)\left[K_{0}(\kappa a)-\varphi K_{2}(\kappa a)\right]+K_{1}(\kappa b)\left[I_{0}(\kappa a)-\varphi I_{2}(\kappa a)\right]}{I_{1}(\kappa b) K_{0}(\kappa a)+K_{1}(\kappa b) I_{0}(\kappa a)}$,

where $b=a \varphi^{-1 / 2}$, but the analytical form of $\left\langle\Theta_{2}\right\rangle$ is quite complicated and it will be determined by numerical integration. Note that, when the symmetric electrolyte is replaced by a general electrolyte, there should be one more term of the order $\zeta^{2}$ in Eqs. (16) and (18) which is proportional to the parameter $\beta$ [10]. Also, when the effect of radial distributions of ions is taken into account for the local induced electric field in Eq. (12), there should be another term of the order $\zeta^{2}$ in these equations which is proportional to $\beta^{2}-1$.

In the limit of $\varphi=0$, Eqs. (17a) and (17b) becomes;

$$
\begin{aligned}
& \Theta_{1}(\varphi=0)=1-\frac{K_{0}(\kappa \rho)}{K_{0}(\kappa a)}, \\
& \Theta_{2}(\varphi=0)=\frac{1}{\left[K_{0}(\kappa a)\right]^{2}}[H(\kappa a)-H(\kappa \rho)],
\end{aligned}
$$

where the function $H$ is defined as:

$$
H(x)=x^{2}\left\{K_{0}(x)\left[K_{0}(x)+K_{2}(x)\right]-2\left[K_{1}(x)\right]^{2}\right\} .
$$

When $\rho / a \rightarrow \infty$, Eqs. (21a) and (21b) reduces to $\Theta_{1}(\varphi=0)=1$ for all values of $\kappa a$ and $\Theta_{2}(\varphi=$ $0)=H(\kappa a) /\left[K_{0}(\kappa a)\right]^{2}$. When $\kappa a=0$, Eqs. (21a) and (21b) gives $\Theta_{1}(\varphi=0)=0$ for all finite values of $\rho / a, \Theta_{2}(\varphi=0)=0$ for all values of $\rho / a$, $\left\langle\Theta_{1}\right\rangle(\varphi=0)=1$, and $\left\langle\Theta_{2}\right\rangle(\varphi=0)=0$.

In the limiting situation that $\kappa a=0$, Eqs. (17a) and (17b) gives $\Theta_{1}=\Theta_{2}=0$ at any position in the cell with a finite value of $\varphi$, which also leads to $\left\langle\Theta_{1}\right\rangle=\left\langle\Theta_{2}\right\rangle=0$, as expected. If we consider the other limiting situation that $\kappa a \rightarrow \infty$, the fluid velocity at a large distance from the surface of the cylinder (i.e. with $\kappa(\rho-a) \rightarrow \infty)$ can be evaluated from Eq. (16) noting that $\psi(\rho) \rightarrow 0$ far from the cylinder. For this particular case, $\Theta_{1}=\Theta_{2}=1$ at an arbitrary position $\rho / a \neq 1$ (so $\left\langle\Theta_{1}\right\rangle=\left\langle\Theta_{2}\right\rangle=$ $1-\varphi$ due to the definition of Eq. (19)) and Eq. (16) is consistent with the result obtained by Prieve et al. [5] for the diffusioosmotic flow of a symmetric electrolyte solution near an infinite flat plane.

It is understood that the diffusioosmosis of an electrolyte solution near a rigid surface results from a linear combination of two effects: (i) 
"chemiosmosis" due to the non-uniform adsorption of counterions in the electric double layer over the charged surface, which is analogous to the diffusioosmosis of a nonelectrolyte solution $[4,8,9]$; (ii) "electroosmosis" due to the macroscopic electric field generated by the concentration gradient of the electrolyte and the difference in mobilities of the cation and anion of the electrolyte, given by Eq. (12). The terms in Eqs. (16) and (18) proportional to $\beta$ (involving the functions $\Theta_{1}$ and $\left\langle\Theta_{1}\right\rangle$ ) represent the contribution from electroosmosis (same as that caused by an external electric field), while the remainder terms (containing the functions $\Theta_{2}$ and $\left\langle\Theta_{2}\right\rangle$ ) are the chemiosmotic component. Note that the direction of the flow caused by electroosmosis is determined by the sign of the product of the parameter $\beta$ and the surface potential $\zeta$, while the fluid flow generated by chemiosmosis for small values of $\zeta$ is always in the direction of decreasing electrolyte concentration.

\section{Results and discussion}

The functions $\Theta_{1}$ and $\Theta_{2}$ given by Eqs. (17a) and (17b) represent dimensionless electroosmotic velocity of a symmetric electrolyte and chemiosmotic velocity of a general electrolyte, respectively, correct to $\mathrm{O}\left(\zeta^{2}\right)$. Their numerical results in the limit of $\varphi=0$ (maximum porosity of the fiber matrix), calculated by using Eqs. (21a) and (21b), are plotted versus the dimensionless coordinate $\rho /$ $a$ in Fig. 2 for various values of $\kappa a$. As expected, for a specified value of $\kappa a$, both $\Theta_{1}$ and $\Theta_{2}$ are monotonically increasing functions of $\rho / a$ from zero at the surface of the cylinder (with $\rho=a$ ) to maxima far away from the cylinder. For a fixed value of $\rho / a$, both $\Theta_{1}$ and $\Theta_{2}$ increase monotonically with an increase in $\kappa a$. Note that $\Theta_{1}(\varphi=$ $0)=1$ as $\rho / a \rightarrow \infty$ means that the electroosmotic velocity of the bulk fluid along the cylinder is given by the Helmholtz formula (equal to $-\varepsilon \zeta E / 4 \pi \eta$ ) regardless of the value of $\kappa a$.

In Fig. 3, the values of $\Theta_{1}$ and $\Theta_{2}$ at the outer boundary of the cell $(\rho=b)$ calculated from Eqs. (17a) and (17b) are plotted versus $\kappa a$ with $\varphi$ as a parameter. It can be seen that both functions $\Theta_{1}$
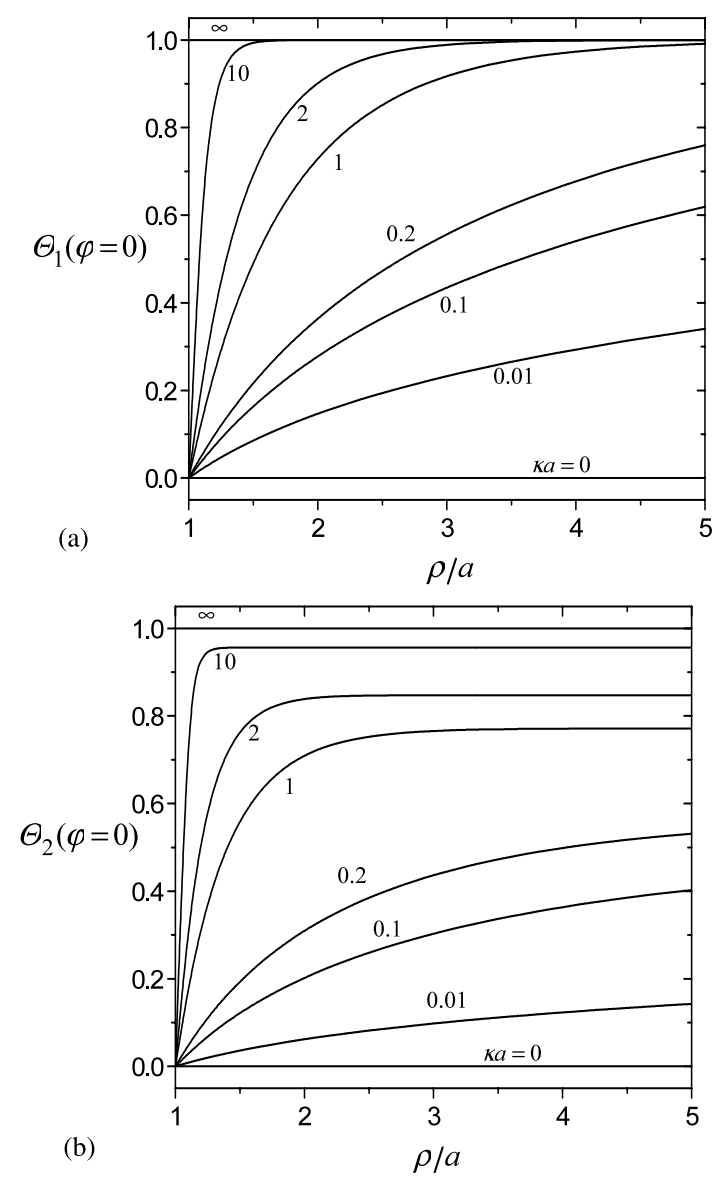

Fig. 2. Plots of the functions $\Theta_{1}(\varphi=0)$ and $\Theta_{2}(\varphi=0)$ as calculated from Eqs. (21a) and (21b) vs. the normalized coordinate $\rho / a$ for various values of $\kappa a$.

and $\Theta_{2}$ are always positive and increase monotonically with the increase of $\kappa a$ (or with the decrease of the double-layer overlap) for a given value of $\varphi$. For a fixed value of $\kappa a$, both $\Theta_{1}$ and $\Theta_{2}$ are monotonically decreasing functions of $\varphi$. The effect of the porosity $(1-\varphi)$ of the fiber matrix on $\Theta_{1}$ and $\Theta_{2}$ can be significant even when the porosity is fairly high.

The numerical results of the averaged $\left\langle\Theta_{1}\right\rangle$ and $\left\langle\Theta_{2}\right\rangle$ as functions of $\varphi$ with $\kappa a$ as a parameter are plotted in Fig. 4. The calculations are presented up to $\varphi=0.9$, which corresponds to the maximum attainable volume fraction (or minimum porosity) for a swarm of identical parallel cylinders [triangularly ordered, [36]]. Again, $\left\langle\Theta_{1}\right\rangle$ and $\left\langle\Theta_{2}\right\rangle$ 

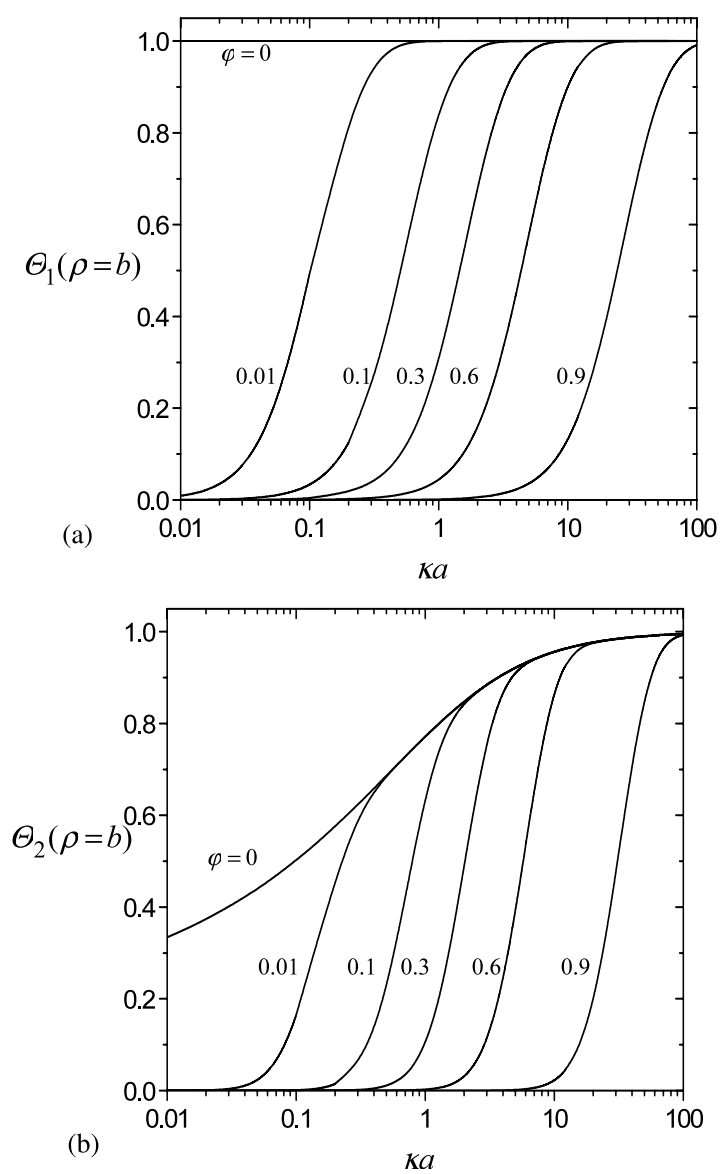

Fig. 3. Plots of the functions $\Theta_{1}(\rho=b)$ and $\Theta_{2}(\rho=b)$ vs. $\kappa a$ with $\varphi$ as a parameter.

increase monotonically with an increase in $\kappa a$ and with a decrease in $\varphi$. As expected, $\left\langle\Theta_{1}\right\rangle$ and $\left\langle\Theta_{2}\right\rangle$ (even $\left\langle\Theta_{1}\right\rangle /(1-\varphi)$ and $\left\langle\Theta_{2}\right\rangle /(1-\varphi)$ ) are smaller than $\Theta_{1}(\rho=b)$ and $\Theta_{2}(\rho=b)$, respectively, for arbitrary finite values of $\kappa a$ and $\varphi$.

Fig. 5 illustrates the dependence of the average diffusioosmotic velocity within an array of identical charged cylinders defined by Eqs. (18) and (19) on their dimensionless surface (zeta) potential at several values of $\kappa a$ and $\varphi$. The magnitude of the fluid velocity is normalized by a characteristic value given by:

$U^{*}=-\frac{\varepsilon\left|\nabla n^{\infty}\right|}{4 \pi \eta n^{\infty}(0)}\left(\frac{k T}{Z e}\right)^{2}$.
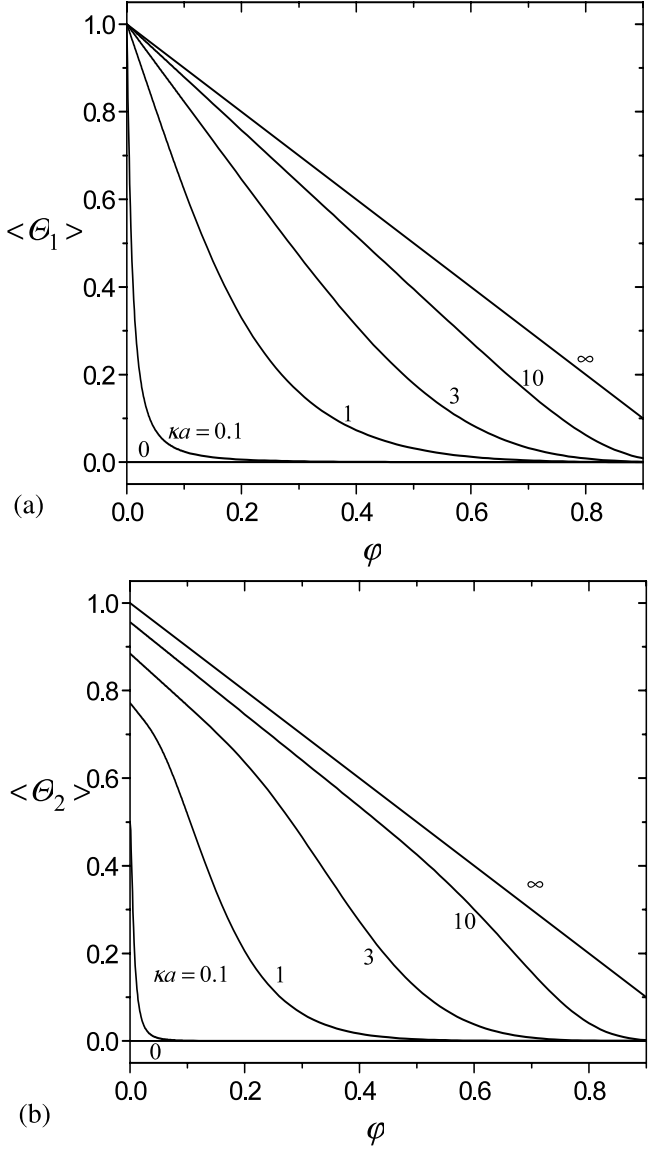

Fig. 4. Plots of the functions $\left\langle\Theta_{1}\right\rangle$ and $\left\langle\Theta_{2}\right\rangle$ vs. $\varphi$ with $\kappa a$ as a parameter.

The negative nature of this characteristic velocity means that its direction is opposite to that of the concentration gradient of the electrolyte. The case that the anion and cation diffusivities are equal $(\beta=0)$ is drawn in Fig. 5(a). Only the results at positive surface potentials are shown because the diffusioosmotic velocity, which is due to the chemiosmotic effect entirely, is an even function of the surface potential $\zeta$ as indicated by Eq. (18). Since our analysis is based on the assumption of small surface potential, the magnitudes of $Z e \zeta / k T$ considered are less than 2. As expected, in this range of $Z e \zeta / k T$, the magnitude of the reduced diffusioosmotic velocity $\langle u\rangle / U^{*}$ (or $u / U^{*}$ at any location) increases monotonically with an increase 

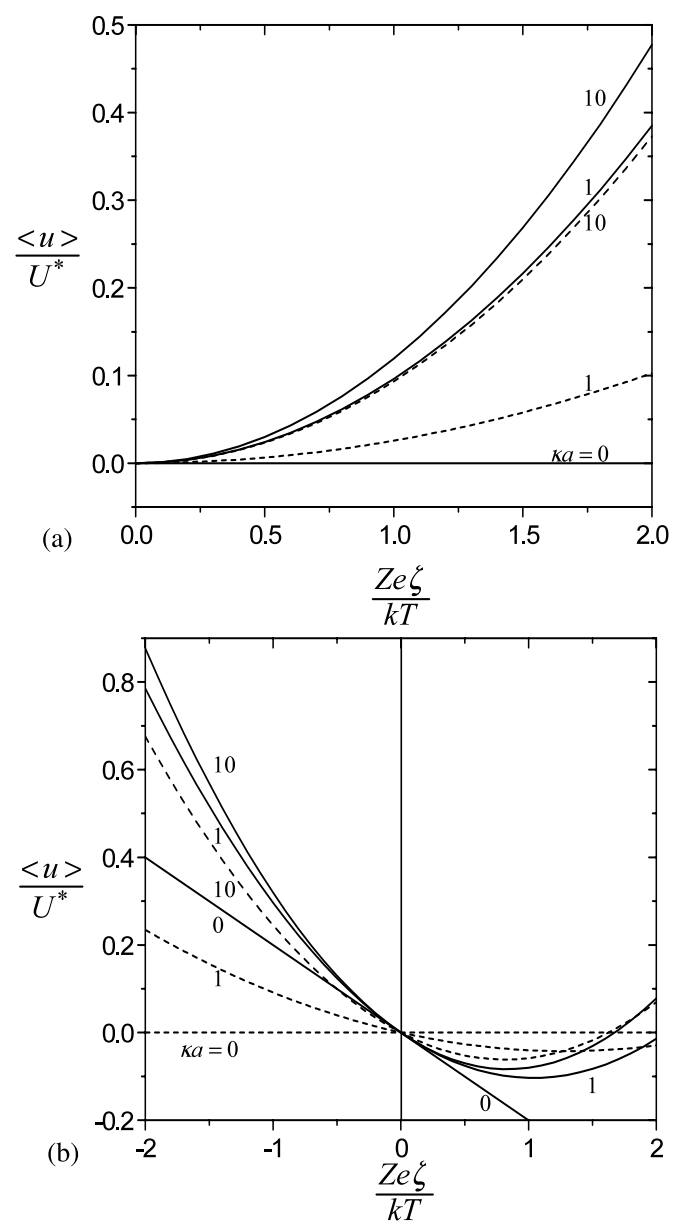

Fig. 5. Plots of the reduced average diffusioosmotic velocity of an electrolyte solution in an ordered array of identical circular cylinders vs. the dimensionless zeta potential of the cylinders at fixed values of $\kappa a$; (a) $\beta=0$; (b) $\beta=-0.2$. The solid and dashed curves represent the cases of $\varphi=0$ and 0.2 , respectively.

in $Z e \zeta / k T$ for given values of $\kappa a$ and $\varphi$. There is no chemiosmotic motion for the special cases of $Z e \zeta / k T=0$ or $\kappa a=0$.

Fig. 5(b) is plotted for the reduced average diffusioosmotic velocity $\langle u\rangle / U^{*}$ of the fluid in the fiber matrix for a case that the cation and anion of the electrolyte have different diffusion coefficients $(\beta=-0.2$ is chosen). In this case, both the chemiosmotic and the electroosmotic effects contribute to the fluid flow and the net diffusioosmotic velocity is neither an even nor an odd function of the surface potential. It can be seen that $\langle u\rangle /$ $U^{*}$ (or $u / U^{*}$ at any location) is not necessarily a monotonic function of $Z e \zeta / k T$ given constant values of $\kappa a$ and $\varphi$. Some of the curves in Fig. 5(b) show that the fluid flow might reverse its direction more than once as the surface potential of the cylinders varies from negative to positive values. The reversals occurring at the values of $Z e \zeta / k T$ other than zero result from the competition between the contributions from chemiosmosis and electroosmosis. In the limit $\kappa a=0$, the diffusioosmotic velocity vanishes for any finite value of $\varphi$, but reduces to the result $\langle u\rangle / U^{*}=\beta Z e \zeta / k T$ (due to electroosmosis only) when the porosity of the fiber matrix approaches unity (with $\varphi=0$ ). Note that the situations associated with Fig. 5(a) and (b) (taking $Z=1$ ) are close to the diffusioosmosis of the aqueous solutions of $\mathrm{KCl}$ and $\mathrm{NaCl}$, respectively.

\section{Concluding remarks}

In this paper, the steady-state diffusioosmosis of a solution of a symmetric electrolyte with a uniform imposed concentration gradient in an ordered array of identical parallel circular cylinders with an arbitrary value of $\kappa a$ is analyzed using the unit cell model. The cylinders may have either a constant surface potential or a constant surface charge density. Solving the linearized PoissonBoltzmann equation and modified Navier-Stokes equation applicable to the system of a cylinder in a unit cell, we have analytically obtained the electrostatic potential distribution and the fluid velocity profile. The fluid flow caused by the existence of the streaming potential induced by the diffusioosmosis is not included. Closed-form formulas and numerical results for the diffusioosmotic and electroosmotic velocities of the electrolyte solution are presented as functions of $\kappa a$ and the porosity of the array of cylinders correct to the order $\sigma^{2}$ or $\zeta^{2}$.

Equation (16) with $\Theta_{1}$ and $\Theta_{2}$ given by Eqs. (21a) and (21b) for the limiting case of $\varphi=0$ and $\rho / a \rightarrow \infty$ can be used to express the diffusiophoretic/electrophoretic velocity (in the opposite direction) of an isolated charged circular cylinder along 
its axis. It is found that this electrophoretic velocity is given by the Smoluchowski formula (equal to $\varepsilon \zeta E / 4 \pi \eta$ ) no matter what the value of $\kappa a$ is. As to the diffusiophoretic motion of a circular cylinder generated by a transverse electrolyte concentration gradient, the velocity of the cylinder has also been obtained [34]. For the diffusiophoresis of a dielectric cylinder oriented arbitrarily with respect to the imposed electrolyte gradient, the cylinder velocity is the vectorial sum of its transversal and longitudinal contributions. For an ensemble of circular cylinders with random orientation, the average diffusiophoretic/electrophoretic velocity (aligned with the direction of $\nabla n^{\infty}$ or the macroscopic electric field) can be obtained by Eq. (16) with $\Theta_{1}$ and $\Theta_{2}$ equal to one third of their values given by Eqs. (21a) and (21b) with $\rho / a \rightarrow \infty$ plus two thirds of their transverse counterparts.

Equations (12) and (16)-(22) are obtained on the basis of the Debye-Huckel approximation for the equilibrium potential distribution around the dielectric cylinder with a low zeta potential in a unit cell. The reduced formula for $\Theta_{1}$ of a single dielectric sphere with low zeta potential in an unbounded electrolyte solution was compared with the general solution and shown to give a good approximation for the case of reasonably high zeta potentials (with errors less than $4 \%$ for $|\zeta| e / k T \leq 2)$ [37]. Also, comparing with the numerical solution for the diffusiophoretic mobility of an isolated charged sphere in $\mathrm{KCl}$ and $\mathrm{NaCl}$ aqueous solutions obtained by Prieve and Roman [38] valid for an arbitrary value of zeta potential, we find that the corresponding result for a dilute suspension of charged spheres with low zeta potential is also quite accurate for the entire range of $|\zeta| e / k T \leq 2$ [39]. Therefore, our results in Eqs. (16), (17a), (17b), (18)-(20), (21a), (21b) and (22) might be used tentatively for the situation of reasonably high electric potentials. In order to see whether our approximate solution can be extended to the higher values of electric potential, a numerical solution of the electrokinetic equations with no assumption on the magnitude of electric potential would be needed to compare it with the approximate solution.

\section{Acknowledgements}

This research was supported by the National Science Council of the Republic of China under Grant NSC90-2214-E-002-019.

\section{References}

[1] M. Smoluchowski, Handbuch der Electrizitat und des Magnetismus (Ed) I. Graetz, vol. II. Barth, Leipzig, 1921, p. 336.

[2] D. Burgreen, F.R. Nakache, J. Phys. Chem. 68 (1964) 1084.

[3] C.L. Rice, R. Whitehead, J. Phys. Chem. 69 (1965) 4017.

[4] S.S. Dukhin, B.V. Derjaguin, in: E. Matijevic (Ed.), Surface and Colloid Science, vol. 7, Wiley, New York, 1974.

[5] H.J. Keh, Y.C. Liu, J. Colloid Interf. Sci. 172 (1995) 222.

[6] H.J. Keh, H.C. Tseng, J. Colloid Interf. Sci. 242 (2001) 450.

[7] D.C. Prieve, J.L. Anderson, J. Ebel, M.E. Lowell, J. Fluid Mech. 148 (1984) 247.

[8] J.L. Aderson, Annu. Rev. Fluid Mech. 21 (1989) 61.

[9] H.J. Keh, S.B. Chen, Langmuir 9 (1993) 1142.

[10] H.J. Keh, J.H. Wu, Langmuir 17 (2001) 4216.

[11] J. Happel, AIChE J. 4 (1958) 197.

[12] J. Happel, AIChE J. 5 (1959) 174.

[13] S. Kuwabara, J. Phys. Soc. Jpn. 14 (1959) 527.

[14] S. Levine, G.H. Neale, J. Colloid Interf. Sci. 47 (1974) 520.

[15] V.N. Shilov, N.I. Zharkikh, Y.B. Borkovskaya, Colloid J. USSR (English Translation) 43 (1981) 434.

[16] N.I. Zharkikh, V.N. Shilov, Colloid J. USSR (English Translation) 43 (1982) 865.

[17] M.W. Kozak, E.J. Davis, J. Colloid Interf. Sci. 127 (1989) 497.

[18] M.W. Kozak, E.J. Davis, J. Colloid Interf. Sci. 129 (1989) 166.

[19] H. Ohshima, J. Colloid Interf. Sci. 188 (1997) 481.

[20] T.J. Johnson, E.J. Davis, J. Colloid Interf. Sci. 215 (1989) 397.

[21] J.M. Ding, H.J. Keh, J. Colloid Interf. Sci. 236 (2001) 180.

[22] F. Carrique, F.J. Arroyo, A.V. Delgado, J. Colloid Interf. Sci. 243 (2001) 351.

[23] H. Ohshima, J. Colloid Interf. Sci. 212 (1999) 443.

[24] S. Levine, G.H. Neale, N. Epstein, J. Colloid Interf. Sci. 57 (1976) 424.

[25] H.J. Keh, J.M. Ding, J. Colloid Interf. Sci. 227 (2000) 540.

[26] J.M. Ding, H.J. Keh, J. Colloid Interf. Sci. 243 (2001) 331.

[27] F. Carrique, F.J. Arroyo, A.V. Delgado, Colloids Surf. A 195 (2001) 157.

[28] H. Ohshima, J. Colloid Interf. Sci. 208 (1998) 295.

[29] N.I. Zharkikh, Y.B. Borkovskaya, Colloid J. USSR (English Translation) 43 (1982) 520. 
[30] M.W. Kozak, E.J. Davis, J. Colloid Interf. Sci. 112 (1986) 403.

[31] H. Ohshima, J. Colloid Interf. Sci. 210 (1999) 397.

[32] E. Lee, Y. Lee, F. Yen, J. Hsu, J. Colloid Interf. Sci. 223 (2000) 223.

[33] Y.K. Wei, H.J. Keh, J. Colloid Interf. Sci. 248 (2002) 76.

[34] H.J. Keh, Y.K. Wei, Langmuir 18 (2002) 10475.
[35] V.G. Levich, Physicochemical Hydrodynamics, Prentice Hall, Englewood Cliffs, NJ, 1962.

[36] J.G. Berryman, Phys. Rev. A 27 (1983) 1053.

[37] R.W. O’Brien, L.R. White, J. Chem. Soc., Faraday Trans. 274 (1978) 1607.

[38] D.C. Prieve, R. Roman, J. Chem. Soc. Faraday Trans. 283 (1987) 1287.

[39] H.J. Keh, Y.K. Wei, Langmuir 16 (2000) 5289. 